\title{
Control of a Four-wire Hybrid Prosumer Converter for Balancing Utility Grids
}

Łukasz Kwaśny*, Dariusz Zieliński

Faculty of Electrical Engineering and Computer Science, Department of Electrical Drives and Machines, Lublin University of Technology, Lublin, Poland

Received: November 04, 2020; Accepted: February 07, 2021

\begin{abstract}
The paper presents the project of the power electronic $\mathrm{AC} / \mathrm{DC}$ converter operating under a multiresonant control algorithm for prosumer applications. This design allows independent control of active and reactive power for any or each phase. Both the converter and its algorithm are based on a three-phase converter of four-wire topology (AC/DC 3p-4w) with a DC bus, which couple the converter to a renewable energy source and energy storage. Further, model and simulation tests were carried out in the Matlab-SIMULINK programming environment. The results obtained indicate that operation with deep unbalances and powers of opposite signs in individual phases results in current variations (oscillations) in the DC line, which is a significant limitation, and it can be concluded that the level of asymmetry should be limited to the level acceptable to the energy storage device.
\end{abstract}

Keywords: control of 4-wire converter • utility grid balancing • prosumer generation • hybrid PV converter • energy storage

\section{Introduction}

Due to the increasing number of electronic devices used in households are equipped with impulse power supplies, more and more often the electricity consumer is considered by the power system to be non-linear. On the other hand, the same consumer becomes an energy producer if he has his own photovoltaic energy source. Thus, he ceases to be a passive energy consumer and becomes an active prosumer (Grijalva et al., 2011; Zielińska et al., 2016) that can positively influence on the quality of energy flowing between the household and the power system. When the prosumer operates on a stiff grid, it is required that the control algorithm of the converter keeps the THD factor of the current below a certain threshold standard. Fulfilment of this criterion may be problematic when grids are saturated with non-linear energy consumers and distributed energy sources that introduce disturbances into the power grid. In addition, a large number of uncontrolled renewable energy sources, especially single-phase generators, may also contribute to significant changes in voltage amplitudes in individual phases of the power system. Therefore, a need arises for developing an algorithm for controlling the converter to ensure that the nonlinear character of own electronic devices and the disturbances coming from the network have a limited impact on the THD of the current generated at the output of the converter. To improve the symmetry of the utility grid, it is recommended that the converter should improve the symmetry of the network phase voltages. To solve these problems, this article aims to find a solution to develop an algorithm for controlling the converter which will be able to meet the desired requirements.

Based on the literature (Daniel and Cyril, 2017; Islam et al., 2018; Piasecki et al., 2011), a solution based on proportional-resonant regulators and band-pass filters, whose task is the selective elimination of higher harmonics and independent power control in individual phases, has been selected for the implementation of these tasks.

This paper analyses the multi-resonant control system and provides a mathematical description of the regulator. Laboratory tests were carried out with the use of a three-level converter and algorithms implemented on the DSP 
platform by means of automatic code generation and the Matlab/SIMULINK package. The research was carried out with a mapping of the converter's operating system with a network characterised by a strong asymmetry in the load of individual phases.

\section{The Problem of Progressive Desymmetrisation of the $\mathrm{nN}$ Distribution Grid}

In some cases, the progressive development of prosumer installations contributes to a significant deterioration in the quality of electricity in the distribution network due to increasing asymmetries between phases. The reason is that most of the prosumer installations currently being installed do not exceed $3 \mathrm{kWp}$, making them single-phase installations in the vast majority of cases. Examples of inverter solutions for cooperation with mains are presented in Table 1.

Currently, low-voltage distribution network operators rarely perform load audits of particular system phases. Because of this reason, there is a lack of information for selecting and installing the converter in an optimal way for the distribution system. In extreme cases, the converters installed in the lowest loaded phase further increase its voltage and asymmetry between lowest loaded phase and the most loaded phase of the utility grid.

The additional problem is the low load on the grid at the time of the highest generation when the sun is at its peak, and majority of household appliances are switched off. This leads to an excessive increase in phase voltages, increase in losses of the $\mathrm{MV} / \mathrm{nN}$ transformer and faster degeneration of the electrical equipment and to the switching off converters in critical states, and thus to the stopping the generation of distributed sources which are connected to a given line.

Currently used single-phase converters do not have the ability to manage power generation. As a standard, they implement the MPPT algorithm - maximal power point tracking of a photovoltaic panel. Such a strategy seems to be beneficial from the prospective economic point of view, as it allows for maximum use of its photovoltaic installation. However, if this strategy leads to an excessive voltage increase and consequently results in the shutdown of the power line, it causes economic losses to both the operator and the prosumer.

Since operators are generally not in a position to directly influence the level of power generated in individual micro-generation systems, they cannot continuously limit the power generated by their own power plants. This problem can be solved by using energy storage cooperating with a prosumer power converter. Such energy storage then acts as a buffer to store the excess energy. This allows the energy consumption and the feeding into the grid to be shifted to more favourable times of the day for the power system. The three-phase four-wire system converter with energy storage is an extension of a single-phase system. Owing to this solution it is possible not only to store energy, but also to redirect the generation to the phase with the lowest voltage, which leads to the reduction of asymmetries between the individual phases. The use of a four-wire converter gives the technical possibility to simultaneously load the phase with the highest voltage and the generation of the phase with the lowest voltage. In such a situation, inverter operation of single phase and rectifier operation of another phase may occur (Figure 6). The above solution is developed in the next section.

\section{Four-Wire Hybrid Converter with Multi-Resonant Regulators}

The equivalent diagram of the hybrid multi-resonant converter (Figure 1) is made up of a DC/DC converter that increases voltage, constituting an interface between photovoltaic panels and the main DC bus and a four-wire DC/ AC converter. Parallel to the output of these converters, an isolated DC/DC converter is connected in the form of two single-phase bridges connected through a medium frequency transformer. This circuit is the interface between

Table 1. Single-phase power grid inverters.

\begin{tabular}{lccccc}
\hline & Power $[\mathrm{kW}]$ & Output current $(\mathrm{A})$ & Voltage PV $(\mathrm{V})$ & $\eta(-)$ & $\cos \varphi(-)$ \\
\hline \hline Fronius & 3 & 13 & $165-550$ & 0.954 & $0.85-1$ \\
SMA & 3 & 16 & $110-500$ & 0.97 & 0.8 \\
Zeversolar & 3 & 15 & $70-520$ & 0.975 & 0.8 \\
\hline
\end{tabular}




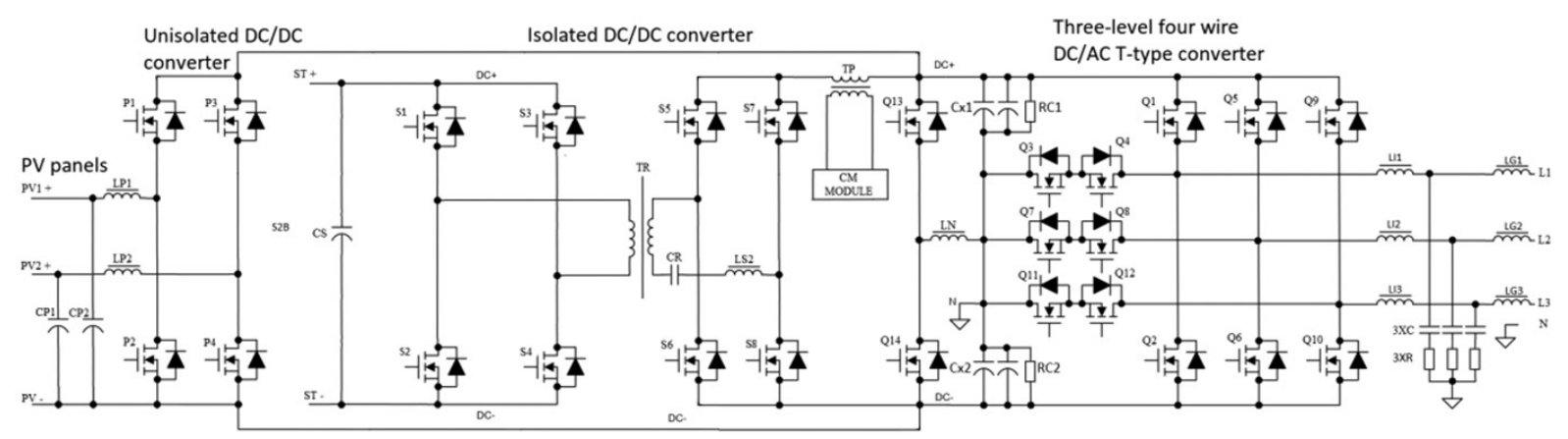

Fig. 1. Hybrid DC/AC converter system in a three-phase four-wire system.

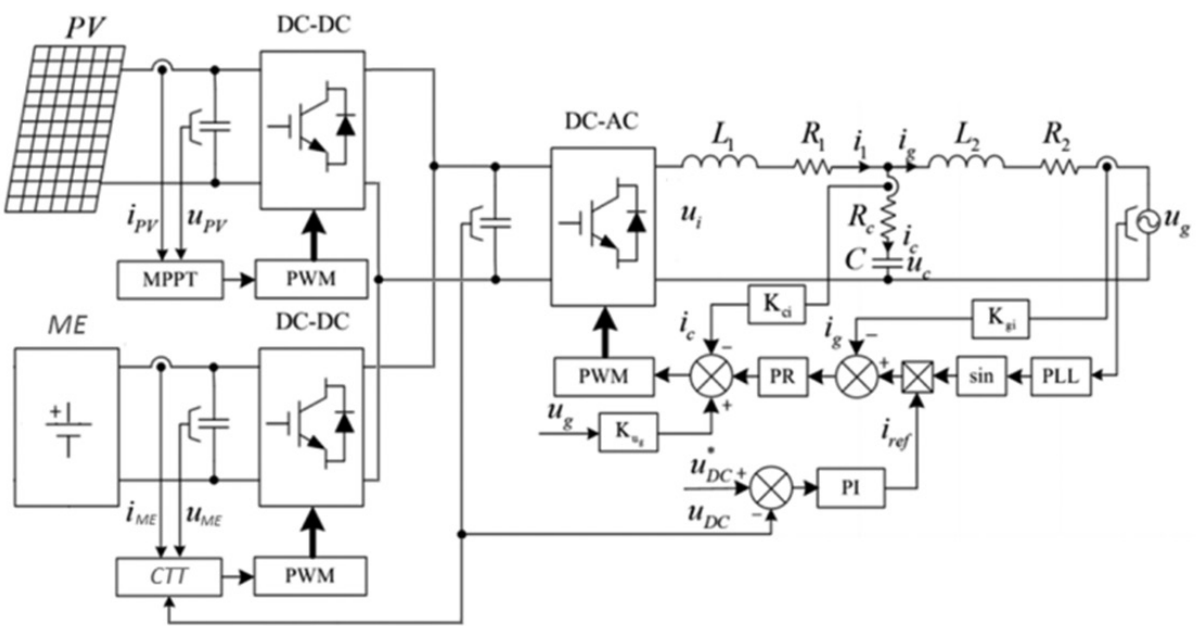

Fig. 2. Simplified algorithm of multiresonant control of the converter for single phase.

the main DC bus of the converter and the electrochemical storage and is connected to the ST+ and ST- terminals. The DC/AC converter is realised as a three-level converter in a T-type circuit (Kouro et al., 2010). Additionally, a fourth wire is introduced, which acts as a neutral wire. The neutral level is realised by dividing the voltage of the DC bus of the converter by using a serial connection of $\mathrm{Cx} 1$ and $\mathrm{C} \times 2$ capacitors. At the same time, the centre of the DC bus is connected by LN inductance to transistors Q13 and Q14. This makes it possible to maintain a constant voltage on the $\mathrm{DC}$ bus centre by discharging the capacity of $\mathrm{Cx} 1$ or $\mathrm{Cx} 2$ using $\mathrm{LN}$ inductance. When the voltage on the Cx2 capacity is increased, the Q14 transistor starts to switching, causing this capacity to be short-circuited by the LN inductance to the negative pole of the DC bus, thus causing it to discharge. At the same time, due to the phenomenon of self-induction, the voltage on $\mathrm{Cx} 1$ capacity increases. By discharging one of the capacitances and boosting the voltage of the other one, there is a quick return to the state of equilibrium - keeping the VDC/2 voltage in the fourth branch of the converter. At the output of the converter, there is a filter whose task is to form a three-level voltage closest to the sine waveform of $50 \mathrm{~Hz}$.

Due to the fact that each phase of the converter is controlled independently, the system was considered as single-phase for further analysis, which was then duplicated three times during simulation-studies.

A system of a hybrid AC/DC converter with energy storage and photovoltaic panel is shown in Figure 2. This figure shows algorithm of multiresonant control for single-phase voltage. To build a three-phase control system with the features presented in Section 2, independent control of each phase was used. Apart from the above, a fourth branch of the converter was introduced, which acts as a neutral wire. The main bus of the AC/DC converter is connected in parallel through independent DC/DC converters with a chain of photovoltaic panels and electrochemical low-voltage energy storage. The unisolated DC/DC converter is connected to PV panels controlled with the MPPT algorithm (Brito et al., 2013). To ensure constant parameters of the main bus voltage of the DC-AC converter, an additional 
Table 2. Hybrid AC/DC converter system parameters

\begin{tabular}{ll}
\hline System output power $(\mathrm{kW})$ & 10 \\
Switching frequency of AC/DC modules $(\mathrm{kHz})$ & 18 \\
Switching frequency of DC/DC modules DC/DC $(\mathrm{kHz})$ & 50 \\
DC bus voltage of the AC/DC converter $(\mathrm{V})$ & 750 \\
Idle voltage of photovoltaic panels $(\mathrm{V})$ & 400 \\
Rated energy storage voltage $(\mathrm{V})$ & 48 \\
Capacity of DC bus ( $\mathrm{mF})$ & 10 \\
Cut-off frequency of the output LCL filter (kHz) & 2,8 \\
LCL filter parameters ( $\mathrm{mH}$ uF $\mathrm{mH})$ & $1.2+5+0.38$ \\
Transistors T-type converter & SK80TMLI12 \\
Transistors high side DAB converter & FF11MR12W1 \\
Transistors low side DAB converter & SK280MB10 \\
THDi (at load above 50\%) (\%) & $<3$ \\
\hline
\end{tabular}

DAB, dual active bridge.

bidirectional isolated DC/DC converter was used, thereby ensuring galvanic separation between the low-voltage energy storage and the power utility grid (Innoue et al., 2007). Such a solution makes the voltage level independent of the energy generated by photovoltaic panels.

The basic system parameters are presented in Table 2.

The DC/DC converter coupled with an energy storage device is controlled as a dual active bridge (DAB) (Barlik et al., 2013). The task of this converter is to keep the main bus voltage of the converter constant by receiving any excess energy which might be generated by the panel and not processed by the DC/AC converter or to supply energy when the energy generated by the photovoltaic panel is insufficient to cover current energy needs. The task of the $\mathrm{DC} / \mathrm{AC}$ converter is to supply the best possible energy to the low voltage distribution network. Due to this reason, an LCL filter is used at the output of the converter and tuned to eliminate interference from switching transistors. To control the active and reactive power at the output of the converter, correct and reliable synchronisation with the distribution grid is required (Blaabjerg et al., 2006). For the purpose of synchronization, it has been proposed to use the DDSRF-PLL algorithm (Jarzyna, 2019; Jarzyna et al., 2020; Nicastri and Nagliero, 2010; Rodriguez et al., 2007; Silva et al., 2004), which operates on the basis of feedback, thereby ensuring that it is able to provide a synchronisation signal with high quality parameters even in case of strong disturbances occurring in the utility grid (Jarzyna et al., 2017). A sawtooth synchronisation signal assuming values in the range $<0,2 \pi>$ is converted into a sine wave reference signal of amplitude 1 . The reference value of the converter current is calculated on the basis of the current voltage of the converter DC bus and is multiplied by the previously generated sine wave signal. The error of the converter current is eliminated to zero by using a proportional-resonant regulator (Teodorescu et al., 2006), whose resonant frequency is equal to the grid frequency. Thus, only the basic harmonic signal is amplified through this method. Due to the presence of higher harmonics in the power grid, a solution of a multi-resonant regulator was proposed, which consists of multiple proportional-resonant regulators connected in parallel. This approach is presented in Figure 3.

The controller's operational transmittance in the system from Figure 3 is described by the following equation from the current deviation input to the output to the converter:

$$
G_{i}=\left(K_{p} \frac{(s+z 0)}{s}+K_{p l_{-} 1 H^{2}}+\sum_{n=1}^{N} \frac{K_{i I_{\_} n H} 2 \omega_{r c l_{\_} n H} s}{s^{2}+2 \omega_{r c l_{-} n H} s+\omega_{O_{-} n H}^{2}}\right) G_{n o t c h} G_{\text {Lead_Lag }}
$$

On the basis of the transfer function Equation (1), the amplitude-phase characteristics of the control system were determined by taking into account the output filter of the converter (Figure 4).

For slow-changing signals, the system has a gain of one, while the gain of the combs at certain frequencies is characteristic of this system. This comb corresponds to the frequencies that are characteristic of the utility grid harmonics: first, third, fifth, seventh and ninth. Above these frequencies, the system is low-pass with an additional 


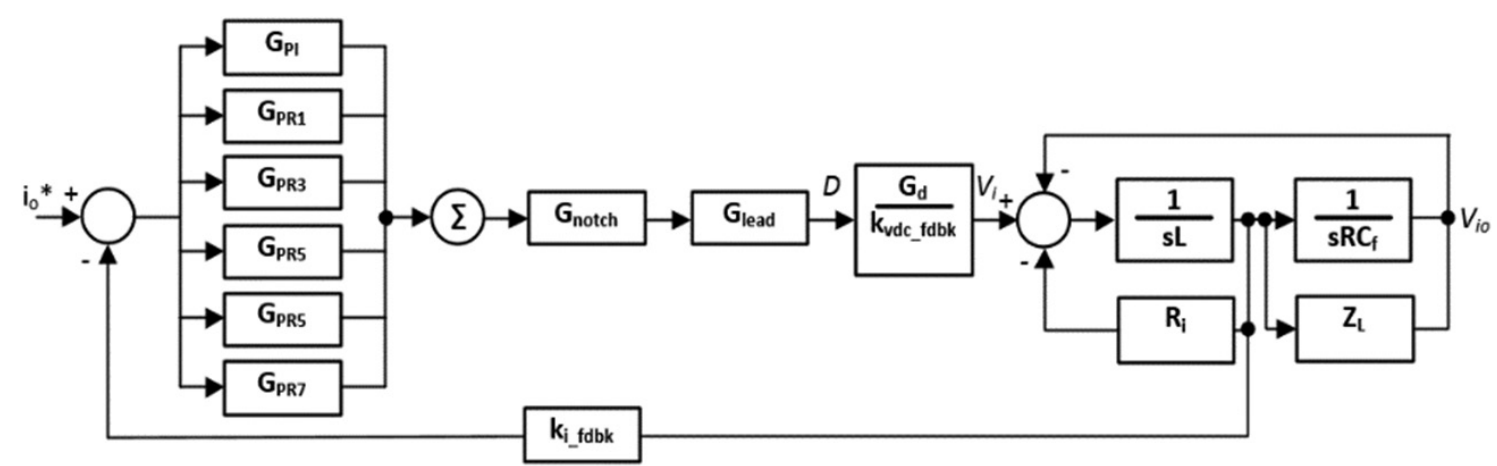

Fig. 3. Transfer function of multi-resonant control of the converter with the following components: GPI - PI regulator; GPR1-7 - proportionally resonant regulators of individual harmonics; Gnotch - notch filter; Glead - lead-lag compensator; G_d/k_(vdc_fdbk) - transformer gain; 1/(sL), 1/(sRC) $R_{i}$ and $Z_{L}$ - individual elements of $L C L$ filter and ki_fdbk - feedback loop.

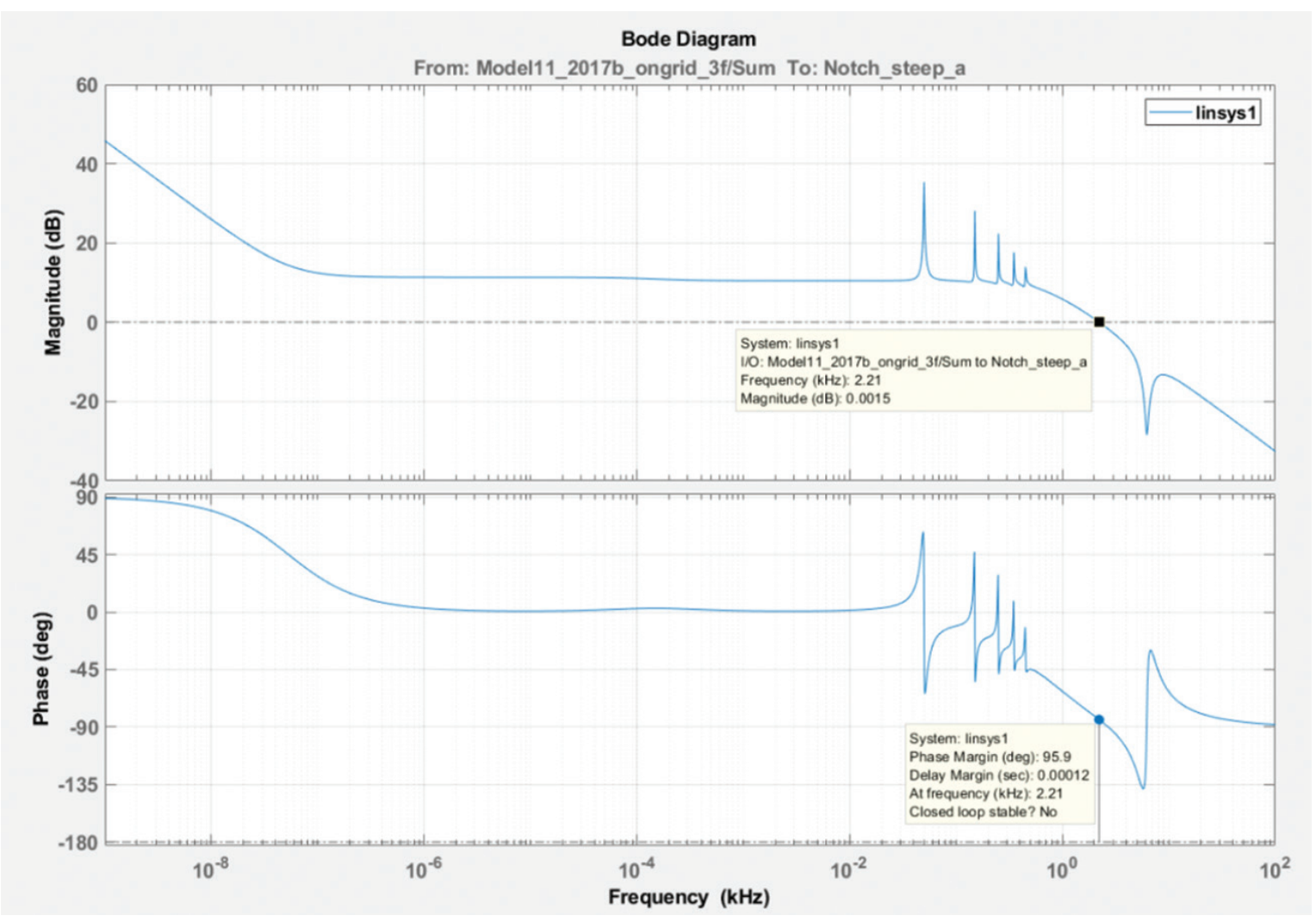

Fig. 4. Amplitude-frequency characteristics of a multiresonant controlled object.

notch filter (Zieliński et al., 2015), which is tuned to eliminate the resonance frequency of the LCL output filter. For simulation tests, the above model has been discretized and implemented in Matlab-Simulink software. Figure 5 shows one of the proportional-resonant regulators applied in the software mentioned. The parameters of the individual regulators have been adopted in such a way as to obtain maximum harmonic amplification for a given frequency, while maintaining an adequate phase reserve. At the same time, the filters are tuned to allow for slight frequency deviation of the frequency range of $3 \mathrm{~Hz}$, while maintaining the gain in this range. It is possible to regard this deviation as a compromise between maximum gain and the margin of error resulting from slight deviations in the frequency of the utility grid.

The proposed control system, depending on the voltage regulation error sign on the main DC bus of the converter, allows for feeding energy to or from the utility grid. The application of multiresonant control together with 


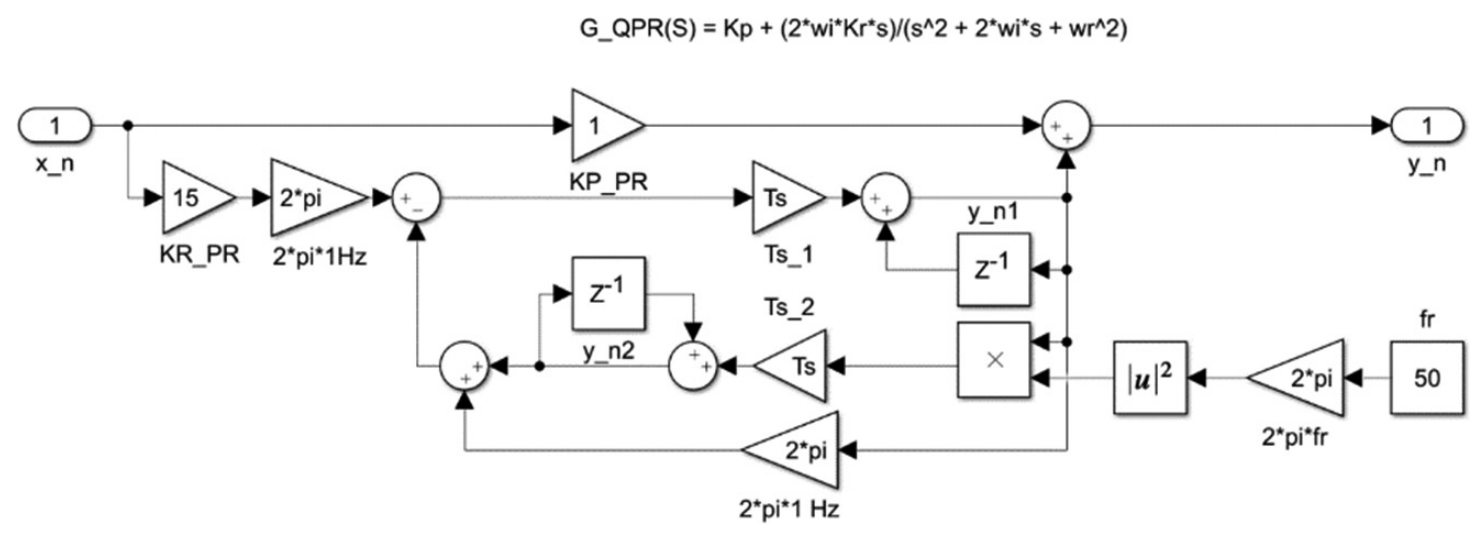

Fig. 5. Digital implementation of the GPR1 resonance transmission algorithm.

Table 3. Table of base parameters of the converter references used during Matlab and SIMULINK simulations.

\begin{tabular}{lll}
\hline Parameter & Type & Value and unit \\
\hline \hline Current reference phase A & Constant & $20(\mathrm{~A})$ \\
Current reference phase B & Triangle wave generator & from -22 to 22 (A) \\
Current reference phase C & Invert triangle wave generator & from 22 to $-22(\mathrm{~A})$ \\
& & $2 \mathrm{~Hz}$ \\
Parameters of simulation & Table 2 & - \\
Grid impedance & Ideal voltage source + Line impedance stabilisation network & According to \\
Control of energy storage DC/DC converter & Maintain constant voltage on the DC bus & EN 55022 \\
Control of AC/DC converter & Controlled current source mode & $750(\mathrm{~V})$ \\
\hline
\end{tabular}

the LCL output filter ensures very good system dynamics, a very small fixed error and THDi at a level below $1 \%$ at a load above $50 \%$ of the rated load of the converter.

\section{Results of Simulation Tests}

The proposed solution in the form of a four-wire converter with multi-resonant controller was implemented in the Matlab/Simulink simulation environment. A study was conducted to determine the possibility of transferring active power in both directions and ensuring a smooth transition between inverter and rectifier operation. Table 3 shows main parameters of the power converter used for the conducted simulation research. Next, the correctness of system operation with independent control of active and reactive power of each phase was tested.

Figure 6 shows the voltage and current waveforms for phase $A$ of a four-wire converter and in this case there was no generation in phases $\mathrm{B}$ and $\mathrm{C}$.

During the test, the power input to the inverter changed as the input to rectifier smoothly as the inverter starts to function as rectifier. Due to the application of multiresonant control, selective elimination of the harmonics (third, fifth, seventh and ninth) was obtained, which consequently has a very high dynamics of the system and high quality of current, which is confirmed by the low THDi coefficient (Figure 7).

The next test was the independent control of active and reactive power of each phase of the converter. During the tests, the situation was such that one of the phases operated in rectifier mode while the other two operated in inverter mode. Also, in this case, two of the phases were changing their mode of operation, and transiting from inverter to rectifier mode.

The consequence of such control is pulsation of DC bus current of the DC/AC converter (Figure 9). These pulsations have a frequency of $100 \mathrm{~Hz}$ and take amplitudes from -0.7 to 1.0 [pu] of the rated inverter current. Such a current pulsation in case of a DC bus with low capacity or lack of energy storage would cause voltage pulsations 


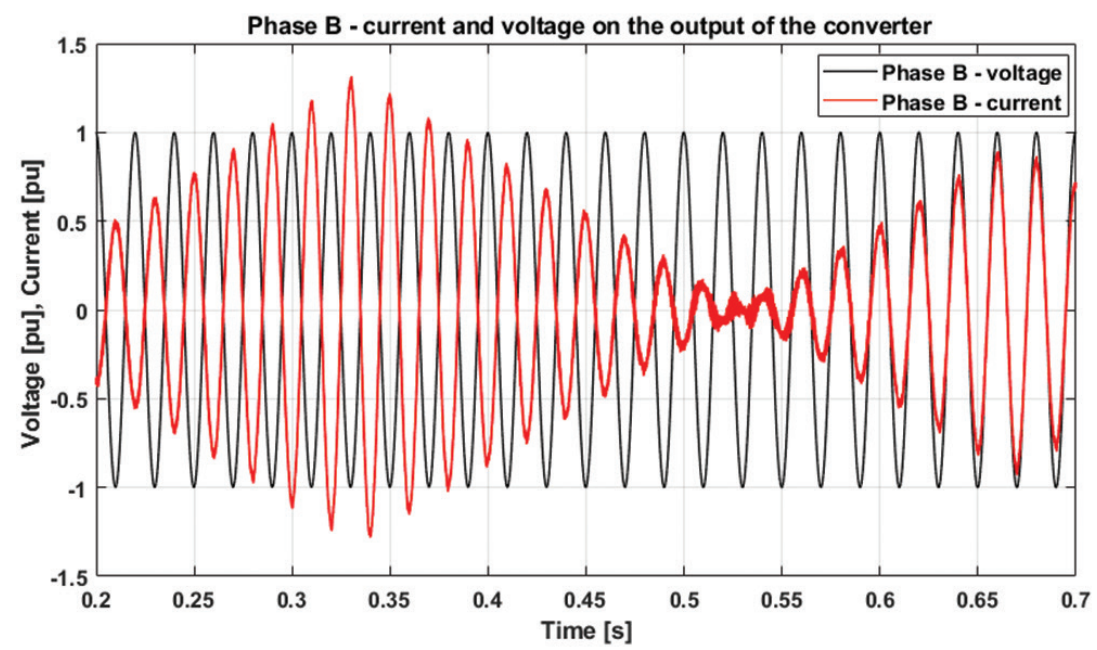

Fig. 6. Conversion from rectifier to inverter $3 p-4 w$ operation (mains mode).
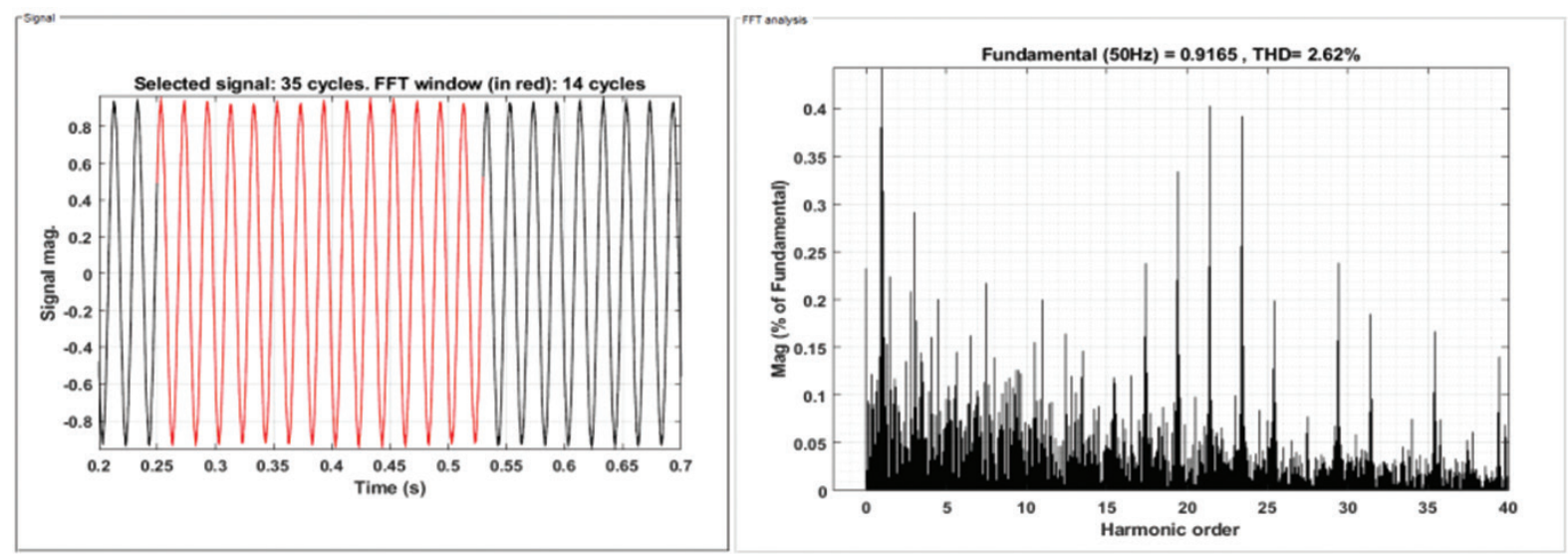

Fig. 7. Analysis of THD current in single phase.

and consequently an increased number of harmonics at the output and unstable operation of the whole converter. Another problem is that the current pulsation is taken over by the energy storage in this model. For electrochemical sources, continuous charging and discharging cycles with a frequency of $100 \mathrm{~Hz}$ are unacceptable, due to the drastic shortening of the life cycle of such storage.

The case discussed in Figure 8 is not a symmetrical one - the sum of currents of individual phases is not equal to zero. It causes the current flow through the neutral wire of the fourth branch of the converter reaching the value of $2.0[\mathrm{pu}]$ of the rated current of the converter (Figure 8).

\section{Laboratory Bench and Commissioning Tests}

The laboratory bench was built using the following functional blocks (Figure 10):

- Main board with signal processor TMS320F28379D

- Three-level, four-wire power module made in T topology

- Energy storage emulating power supply ITECH IT6012-C

- Three isolation transformers

- Four-wire LCL filter with EMC anti-interference module 

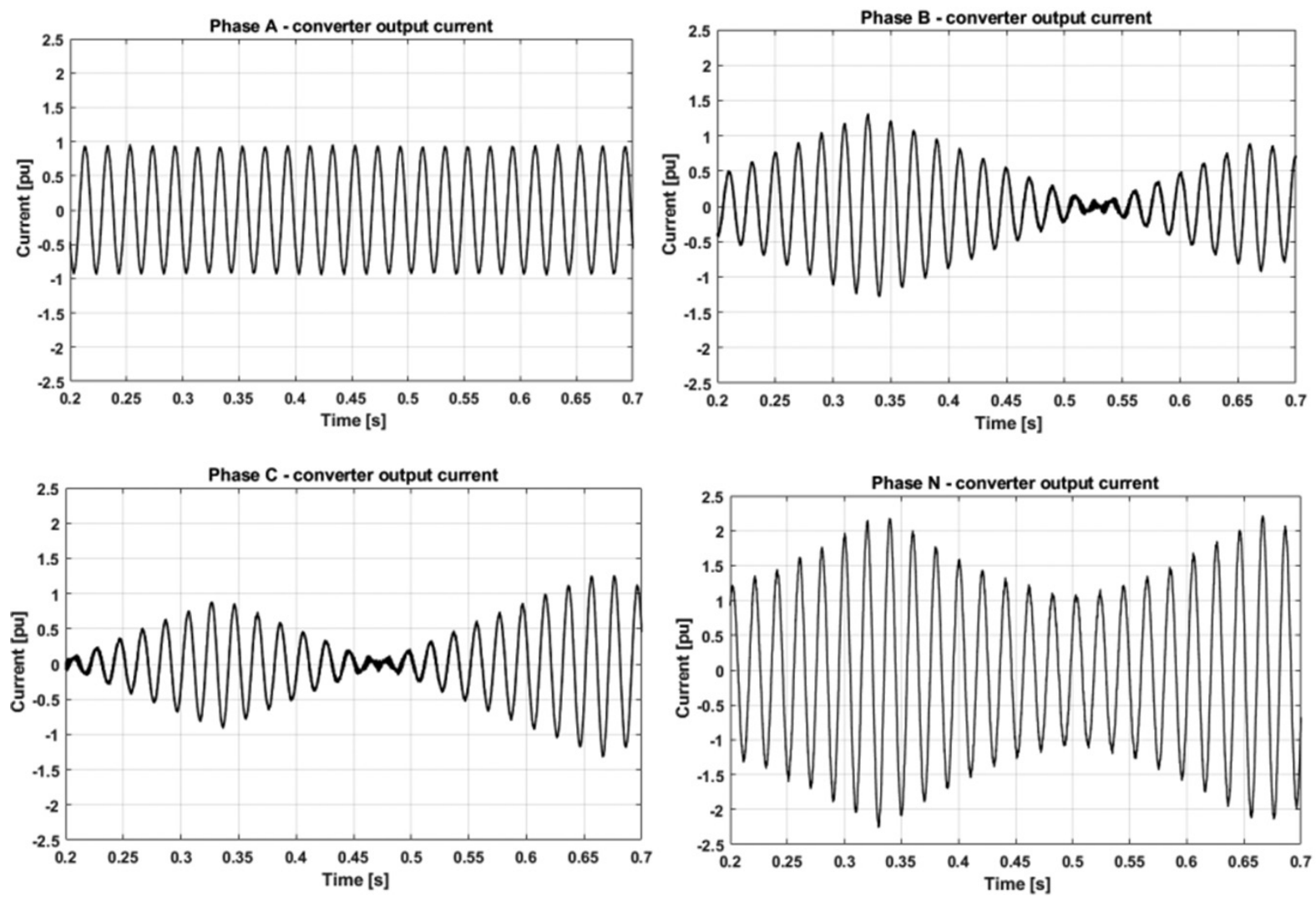

Fig. 8. Independent power control in all phases of the 3p-4w converter (mains mode).

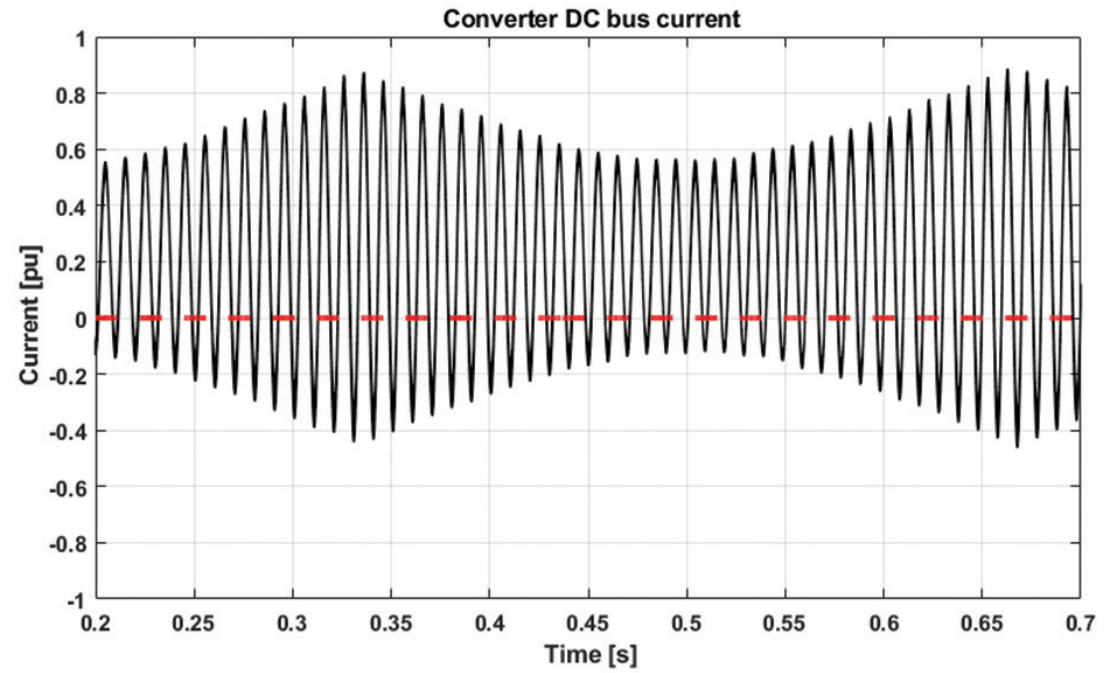

Fig. 9. The DC bus current is alternating, pulsating at $100 \mathrm{~Hz}$.

The motherboard module with the converter was developed for laboratory testing.

Since there is a danger of damaging the lithium-ion storage due to the generation of $100 \mathrm{~Hz}$ current pulsation on the DC bus, a bidirectional power supply IT6012-C with energy storage emulation function was used as a battery simulator. This solution made it possible to carry out tests in different states of asymmetry and differential operation 

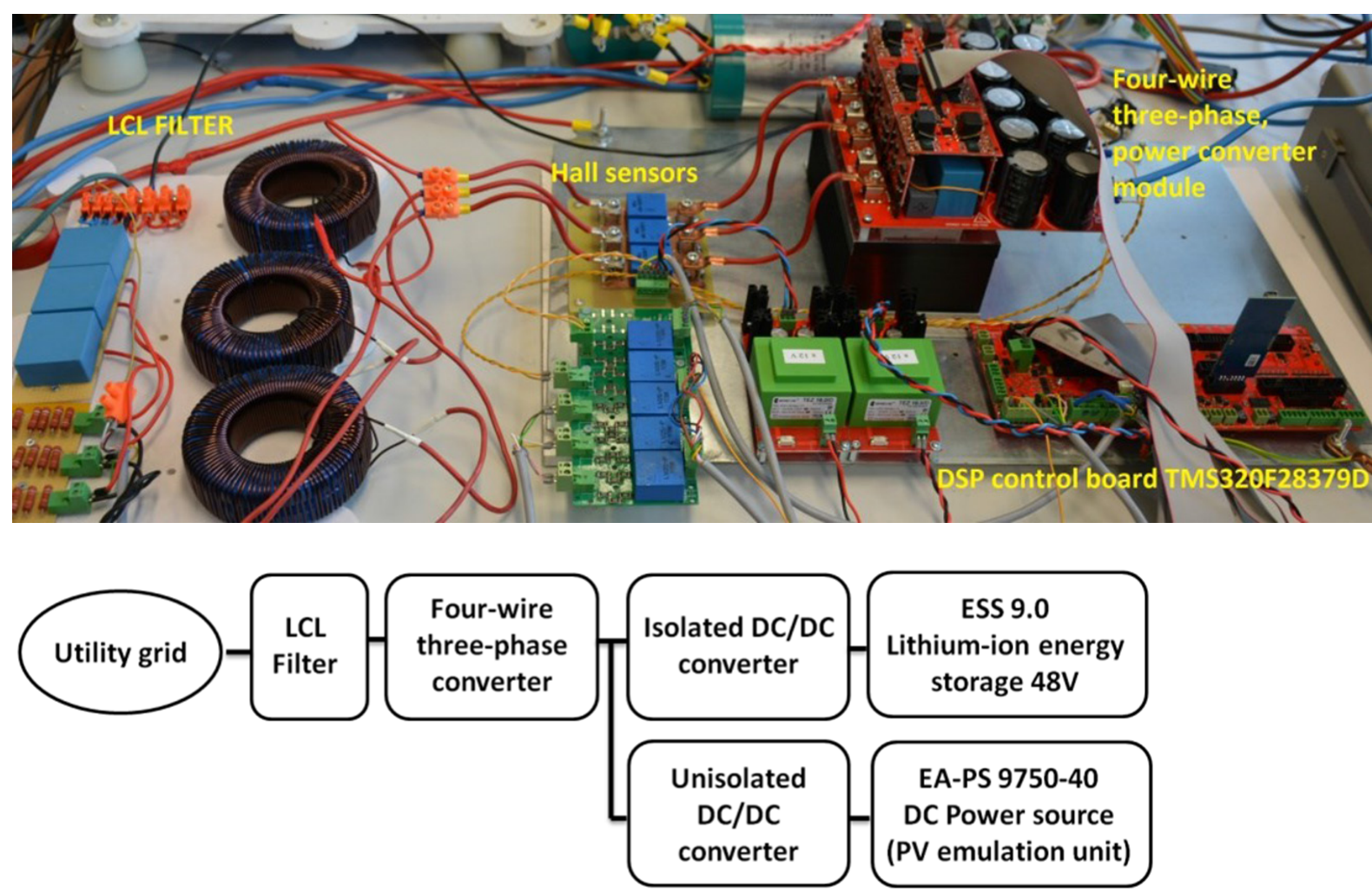

Fig. 10. Converter module used during laboratory tests
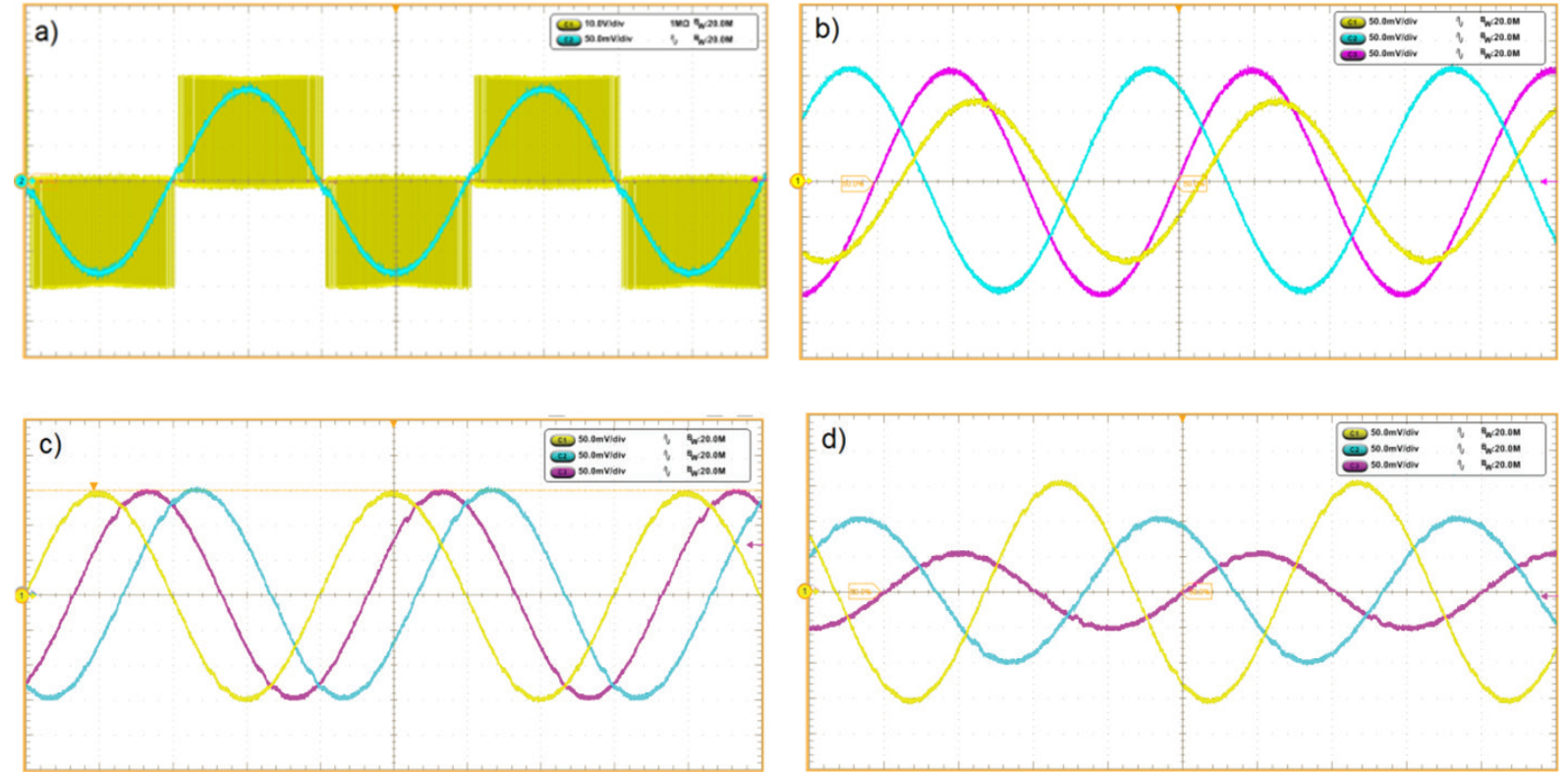

Fig. 11. Oscillograms from the actual operation of the converter: (a) current and voltage on the first phase of the converter, (b) independent current regulation in individual phases of the converter, where current reference value is (Phase: $A+20 A, B+20 A, C 16 A-r e a c t i v e)$, (c) independent current regulation (Phase: $A+20 A, B-20 A, C+20 A$ ), (d) independent current regulation (Phase: $A+15 A, B+10 A, C+20 A$ )

of AC/DC converter phases. The results of laboratory tests (Figure 11) confirmed all the results predicted during the simulation, which approves the full correctness of the model and simulation tests. Further tests in this area are underway and their results will be published in subsequent articles. 


\section{Summary}

The article presents the problem of symmetry of $\mathrm{nN}$ distribution networks with the use of distributed generation sources. Market analysis has shown that most prosumer installations are single-phase. Moreover, it was found that the lack of recording of loads at each of phases of low-voltage grids causes deterioration of power supply conditions in distribution grids of renewable installations. The article presents a solution to the problem in the form of a fourwire converter equipped with an energy storage. Also, a digital implementation of the multiresonant algorithms is proposed as a control system. The validity of the applied algorithms was confirmed by simulation and laboratory tests. Owing to the algorithms, for eliminating individual harmonics of low voltage grids, a system with a very low THDi coefficient of less than $0.83 \%$ for loads above $50 \%$ of the rated load was obtained. During the conducted tests it was concluded that there is a possibility of independent control of reactive and active power in each phase of the proposed converter thanks to the applied multiresonant control algorithms. Unfortunately, such a control strategy has consequences that do not exist for three-phase symmetrical systems. The first disadvantage of the proposed system is the flow of energy through a neutral wire when the sum of the currents of individual phases is not equal to zero.

The article presents an extreme case of generating an active power in two phases in opposite directions. For this case, the maximum current of the neutral wire is equal to twice the rated current of the converter. This case is not a problem for power electronic components because the use of transistors in the neutral wire with increased current capacity is not a strong barrier. A more serious problem for these conditions is the appearance of current pulsation on the DC bus of the converter. This situation is dangerous for the energy storing devices connected to the DC circuit. It has been found that prolonged operation of such an energy store with pulsating current leads to accelerated degradation of this electrochemical source. The efficiency of such a storage device may also be reduced because the electrochemical processes taking place in the lithium-ion cell are encumbered with inertia; thus, the demonstrated rapid changes of current cause the release of large amounts of heat as a result of additional losses in batteries. A potential solution requiring further testing is the use of an additional parallel DC/DC converter with a buffer in the form of a super capacitor. Unfortunately, such a solution significantly complicates the system and increases the total cost of prosumer installation. Nevertheless, the present solution is an alternative to current, uncontrolled prosumer systems and in the future, due to the large number of distributed generation sources, it may become necessary to apply it to maintain symmetry in low voltage distribution networks.

\section{Acknowledgements}

This work was supported under the project 'Management of low-voltage distribution network operation with prosumers' active participation' of the Polish National Centre for Research and Development, Project No. POIR.04.01.02-000007/17, European Funds of the Smart Growth Operational Programme 2014-2020.

\section{References}

Barlik, R., Nowak, M. and Grzejszczak, P. (2013). Power Transfer Analysis in A Single Phase Dual Active Bridge. Bulletin of the Polish Academy of Sciences: Technical Sciences, 61(4), pp. 809-828.

Blaabjerg, F., Teodorescu, R., Liserre, M. and Timbus, A. V. (2006). Overview of Control and Grid Synchronization for Distributed Power Generation Systems. IEEE Transactions on Industrial Electronics, 53(5), pp. 1398-1409.

Brito, M. A. G., Galotto, L., Sampaio, L. P., Melo, G. A. and Canesin, C. A. (2013). Evaluation of the Main MPPT Techniques for Photovoltaic Applications.
IEEE Transactions on Industrial Electronics, 60(3), pp. 1156-1167.

Daniel, Z. and Cyril, S. S. (2017). Design of PR Current Control with Selective Harmonic Compensators Using MATLAB. Journal of Electrical Systems and Information Technology, 4, pp. 347-358.

Grijalva, S., Costley, M. and Ainsworth, N. (2011). Prosumer-Based Control Architecture for the Future Electricity Grid. IEEE International Conference on Control Applications, Denver, pp. 28-30.

Innoue, S. and Akagi, H. (2007). A Bidirectional DCDC Converter for an Energy Storage System with 
Galvanic Isolation. IEEE Transactions on Power Electronics, 22(6), pp. 2299-2306.

Islam, S., Zeb, K., Din, W. and Khan, I. (2018). Design of a Proportional Resonant Controller with Resonant Harmonic Compensator and Fault Ride Trough Strategies for a Grid-Connected Photovoltaic System. Electronics, 7(12), pp. 451.

Jarzyna, W. (2019). A Survey of the Synchronization Process of Synchronous Generators and Power Electronic Converters. Bulletin of the Polish Academy of Sciences. Technical Sciences, 67(6), pp. 1069-1083.

Jarzyna, W., Zieliński, D. and Gopakumar, K. (2020). An Evaluation of the Accuracy of Inverter Sync Angle During the Grid's Disturbances. Metrology and Measurement Systems, 27(2), pp. 355-371.

Jarzyna, W., Zieliński, D., Zielińska, K. and Fatyga, K. (2017). Reduction of Voltage and Power Oscillation in the Two-Phase Shorting of A Grid Inverter. In: 19th European Conference on Power Electronics and Applications (EPE'17 ECCE Europe), Warsaw, 11-14 September 2017.

Kouro, S., Malinowski, M., Gopakumar, K., Pou, J., Franquelo, L. G., Wu, B., Rodriguez, J., Perez, M. A., Leon, J. I. (2010). Recent Advances and Industrial Applications of Multilevel Converters. In: IEEE Transactions on Industrial Electronics, 57(8), pp. 2553-2580.

Nicastri, A. and Nagliero, A. (2010). Comparison and Evaluation of the PLL Techniques for the Design of the Grid-Connected Inverter Systems. In: IEEE International Symposium on Industrial Electronics, Bari, 4-7 July 2010.
Piasecki, S., Jasiński, M. and Milicua, A. (2011). Brief View on Control of Grid-Interfacing AC-DC-AC Converter and Active Filter under Unbalanced and Distorted Voltage Conditions. In: International Journal for Computation and Mathematics in Electrical and Electronic Engineering (COMPEL) on EVER'09, Emerald, 30(1), pp. 351-373.

Rodriguez, P., Pou, J., Bergras, J., Candela, J. I., Burgos, R. P. and Boroyevich, D. (2007). Decoupled Double Synchronous Reference Frame PLL for Power Converters Control. IEEE Transactions on Power Electronics, 22(2), pp. 584-592.

Silva, S. M., Lopes, B. M., Filho, B. J. C., Campana, R. P. and Bosventura, W. C. (2004). Performance Evaluation of PLL Algorithms for Single-Phase Grid-Connected Systems. In: Conference Record of the 2004 IEEE Industry Applications Conference, 2004. 39th IAS Annual Meeting, vol. 4, Seattle, 3-7 October 2004.

Teodorescu, R., Blaabjerg, F., Liserre, M. and Loh, P. C. (2006). Proportional-Resonant Controllers and Filters for Grid-Connected Voltage-Source Converters, IEEE Proceedings-Electric Power Applications, 153(5), pp. 750-762.

Zielińska, K. and Zieliński, D. (2016). Influence of Power Converters on InCreasing the Share of Renewable Energy Sources. Acta Energetica, 1(26), pp. 125-131.

Zieliński, D., Lipnicki, P. and Jarzyna, W. (2015). Synchronization of Voltage Frequency Converters with the Grid in the Presence of Notching. COMPEL - International Journal for Computation and Mathematics in Electrical and Electronic Engineering, 34(3), pp. 657-673. 